\title{
Prompt Gamma Activation Analysis (PGAA) and Short-Lived Neutron Activation Analysis (NAA) Applied to the Characterization of Legacy Materials
}

\author{
Gerald A. English, Richard B. Firestone, Dale L. Perry, Jani P. Reijonen, Ka-Ngo Leung \\ Lawrence Berkeley National Laboratory, Berkeley, CA 94720 (USA) \\ Glenn F. Garabedian \\ Lawrence Livermore National Laboratory, Livermore, CA 94550 (USA) \\ Gabor L. Molnar and Zsolt Revay \\ Institute of Isotope and Surface Chemistry, Budapest H-1525, Hungary
}

Without quality historical records that provide the compositions of legacy materials, the elemental and/or chemical characterization of such materials requires a manual analytical strategy that may expose the analyst to unknown toxicological hazards. In addition, much of the existing legacy inventory also incorporates radioactivity, and, although radiological composition may be determined by various nuclearanalytical methods, most importantly, gamma spectroscopy, current methods of chemical characterization still require direct sample manipulation, thereby presenting special problems with broad implications for both the analyst and the environment. Alternately, Prompt Gamma Activation Analysis (PGAA) provides a 'single-shot', in-situ, non-destructive method that provides a complete assay of all major entrained elemental constituents. Also, it generally obviates the need for sample handling and, thus, minimizes potential exposure to hazardous materials to both people and the environment. Additionally, neutron activation analysis (NAA) using short-lived activation products compliments PGAA, and is especially applicable when the NAA activation surpasses the PGAA in elemental sensitivity. The Lawrence Berkeley National Laboratory (LBNL) has developed a new miniature deuterium-deuterium (D+D) neutron generator that currently outputs $\sim 4 \times 10^{9}$ neutrons/second. It is self-replenishing and low power and maximizes the use of monatomic $\mathrm{D}^{+}$ions that, in turn, maximizes neutron intensity. The LBNL PGAA system also uses a recently updated prompt gamma library database and Hypermet software to convert the raw PGAA spectral data to accurate and precise qualitative and quantitative elemental results. This paper 
discusses the use of PGAA and short-lived NAA to characterize legacy materials at the Lawrence Berkeley National Laboratory (LBNL).

\section{Introduction}

Much of the legacy inventory at LBNL lacks quality historical records that satisfy the pertinent regulatory requirements for full characterization. Most of the inventoried materials also include one or more radioactive constituents, typically actinides, adding another dimension to the overall problem. The radioanalytical problem may generally be addressed by gamma spectroscopy. However, except for the primordial radioactivities with extremely long half-lives like $\mathrm{U}$ and $\mathrm{Th}$, the presence of a visible mass of material is not generally attributable to the radioactive constituents but, instead, to the presence of stable carrier chemicals that must likewise be characterized. In the special case of the actinides, one may glean some information about the cationic moiety of the carrier substrate by alpha-induced x-ray fluorescence since most carrier compounds at LBNL have cationic entities of relatively high $\mathrm{Z}$, thereby affording easier detection and evaluation of the fluorescence $\mathrm{X}$-rays. However, for the anionic moiety, the respective $\mathrm{Z}$ values are usually much lower (e.g., fluorides, nitrates, oxides) and not generally amenable to fluorescence analysis. Therefore, to characterize stable carriers in legacy materials, LBNL needs to identify a methodology that provides the elemental/chemical identity and quantitative composition with minimum sample handling.

In view of these requirements, LBNL has focussed upon PGAA, a technique that is an excitation process not a radioactive-decay process, although PGAA may produce radionuclides by activation during the excitation process. The LBNL system consists of a D+D neutron generator that utilizes an updated database of 35,000 prompt gamma rays and associated Hypermet analytical software to generate highquality elemental analyses of legacy materials. The following sections will discuss the LBNL process in detail.

\section{Experimental}

The analysis of LBNL legacy materials, whether radioactive and/or non-radioactive, requires the use of a high-efficiency, n-type, high-purity germanium (HPGe) detector/multichannel analyzer (MCA) system, 
with a calibrated energy range of $\sim 5 \mathrm{keV}$ to $\sim 2 \mathrm{MeV}$ for NAA decay gammas or to $\sim 11 \mathrm{MeV}^{(1)}$ for PGAA. An n-type detector is preferred due to its relative insensitivity to neutron damage compared with a p-type semiconductor detector ${ }^{(2)}$. To maximize signal intensity, the system must be configured to maximize the number of thermal or 'cold' - those further slowed from thermal energies $(\sim 0.025 \mathrm{eV}$ or $\sim 2200$ meters/second) - neutrons on target. Also, the detector orientation relative to the linear axis of the neutron generator and to the target itself must be optimized. Around the detector, appropriate neutronabsorption materials may be installed between it and the neutron generator to minimize background and the potential for neutron damage. If the detector is positioned as close as possible to the target without compromising protection afforded by the shielding materials, the efficiency of photon collection is maximized, thereby improving overall analytical sensitivity. To this end, the detector should be positioned between $\sim 90^{\circ}$ and $\sim 120^{\circ}$ relative to the linear axis of the neutron generator. To protect against neutron damage to the detector, the neutron generator can be wrapped with a pliable sheath of either ${ }^{6} \mathrm{LiF}$ - or ${ }^{10} \mathrm{~B}_{4} \mathrm{C}$ impregnated silicone rubber. The stable ${ }^{6} \mathrm{Li}$ isotope, $\sim 7.5 \%$ of natural $\mathrm{Li}$, has a high cross-section for absorbing most thermal neutrons with negligible prompt gamma yield ${ }^{(2)}$ by the high cross section $(940 \mathrm{~b})$ nuclear reaction ${ }^{6} \mathrm{Li}(\mathrm{n}, \alpha)^{3} \mathrm{H}$. Also, because the ${ }^{6} \mathrm{Li}$ isotope has such a high reaction cross-section, relatively little $\mathrm{LiF}$ would be required in the sheathing material. Consequently, the contribution of the $\mathrm{F}$ to the background should be negligible with a single activation peak at $~ 1633.6 \mathrm{keV}(100 \%)$ from the formation of radioactive ${ }^{20} \mathrm{~F}\left(\mathrm{t}_{1 / 2} \sim 11.03 \text { seconds }\right)^{(3)}$. At the expected thermal neutron flux of $10^{6} \mathrm{n} \mathrm{cm}^{-1} \mathrm{~s}^{-1},<100 \mathrm{mCi}$ of tritium can be produced from the ${ }^{6} \mathrm{Li}$ reaction which is not a regulatory problem. The availability of large quantities of ${ }^{6} \mathrm{Li}$ is restricted, and $\mathrm{B}_{4} \mathrm{C}$ could be used instead, provided that the single prompt gamma from the ${ }^{10} \mathrm{~B}$ neutron capture reaction, ${ }^{10} \mathrm{~B}(\mathrm{n}, \alpha){ }^{7} \mathrm{Li}$, at $477.6 \mathrm{keV}{ }^{(3)}$ is not a problem. Neutron moderator material would be placed in the forward direction between the neutron source and the target (i.e., $\sim 180^{\circ}$ relative to the linear axis of the neutron generator) to reduce the $2.5 \mathrm{MeV} \mathrm{D}+\mathrm{D}$ neutrons to thermal energies.

2.5 MeV neutrons have been generated by a new upgraded LBNL-developed, self-replenishing, low power ( 600 watts on target), deuterium-deuterium (i.e., D+D)-based neutron generator $\left(\sim 4 \times 10^{9}\right.$ neutrons/second, isotropic). The design of the neutron generator incorporates a novel feature that optimizes the physics of the nuclear-reaction process to maximize neutron yield by producing a preponderant number of monatomic bombarding deuterium ions (i.e., $\mathrm{D}^{+}$). Past attempts at developing a high intensity D+D 
neutron generator had achieved minimal success generally due to an inability to eliminate the overwhelming presence of the countervailing polyatomic deuterium ions (i.e., $\mathrm{D}_{\mathrm{x}}^{+}$) that are very inefficient at producing neutrons. The $\mathrm{D}+\mathrm{D}$ modality also has the added benefit of not using or generating tritium with its attendant regulatory and environmental implications. Polyethylene has been used as the moderator to produce thermal neutrons.

The prompt gamma library consists of 35,000 literature-based gamma-ray energies and thermal cross sections representing all isotopes from ${ }^{1} \mathrm{H}$ to ${ }^{238} \mathrm{U}$, inclusive, where the relative cross section yields have been normalized to precise measurements obtained for all elements at the Budapest reactor. The PGAA library data from all reliable sources were consequently merged into the final database. This updated library was developed by LBNL under the aegis of an International Atomic Energy Agency (IAEA) Coordinated Research Project (CRP) and is superior to earlier databases that were developed in the 1960 's ${ }^{(4)}$. PGAA is an accepted mainstream analytical process in use at the National Institute for Standards and Technology (NIST), but the quality of the requisite database must be high. Thus, the new IAEAsponsored database was developed and is used in the LBNL work.

To establish neutron-generator based PGAA as a high-quality (i.e., accurate and precise) qualitative and quantitative methodology for elemental and/or chemical assay, initial experiments must be conducted in a comparative mode where relevant PGAA results are compared with results obtained by employing alternative, accepted, analytical methodologies. Examples of such methodologies are wet-chemical or instrumental processes, or, if relevant primary standards or high-purity chemicals exist which have documented assays establishing the entrained quality, such materials may accordingly be used. With specific respect to the use of the upgraded LBNL neutron generator in the PGAA process, certain results would be obtained with this generator and compared with PGAA results obtained on similar samples at the Budapest Reactor PGAA Facility that has an established high-quality track record on PGAA. In this manner, the quality of the results for PGAA using the LBNL neutron generator would be further enhanced.

\section{Results and Discussion}

The focus of the LBNL PGAA research to date ${ }^{(5,6,7)}$ has been to determine the requirements for producing high-quality (i.e., accurate and precise) qualitative and quantitative results for in-situ, non- 
destructive assay of legacy materials. Since much of the LBNL legacy inventory consists of radioactivity incorporated into a stable carrier matrix, initial experiments were configured to test the utility of PGAA to account for all entrained carrier species and to determine if chemical stoichiometry may be deduced. Under normal circumstances, characterization of legacy materials would consist of two procedural steps that may be combined into one step due to the fact that both steps involve gamma analysis. If real-time analysis of a radioactive legacy item is conducted under thermal neutron impingement, a composite gamma spectrum is obtained where both the radioactive decay and PGAA lines are collected.

As a first step toward establishing the extent to which PGAA may be used in the characterization of legacy materials, an experiment was conducted at the Budapest Reactor PGAA Facility on a synthetic sample that reflects expected carrier matrices. Accordingly, a sample consisting of non-certified $\mathrm{CaF}_{2}, \mathrm{CeF}_{4}$ and $\mathrm{CeCl}_{3}\left(\mathrm{H}_{2} \mathrm{O}\right)_{\mathrm{x}}$ was initially selected for analysis to determine the capability of PGAA to detect all elemental constituents including impurities. The data are provided in Tables 1 and 2. In Table 1, the ratio of atom $\%$ for hydrogen and oxygen ( $\equiv 2: 1$ ) clearly correlate with waters-of-hydration. Also, from this Table, it is clear that PGAA provides a comprehensive picture of all chemical constituents including impurities. This observation provides strong evidence that PGAA is a potentially inexpensive way to assay a sample in entirety. Other methods would require a hit-or-miss approach that would be both time-consuming and expensive. Table 2 provides a comparative analysis between the actual $\%$ by mass of the mixture as determined by the weighing process in the laboratory and the PGAA data obtained on an aliquot. These data indicate for simple inorganic matrices that not only may elemental signatures be determined but also possibly chemical stoichiometry. The $\mathrm{Ca}$ and $\mathrm{F}$ results are in excellent agreement. The $\mathrm{Ce}$ and $\mathrm{Cl}$ results do not appear to agree as well but that observation may actually be deceiving. As mentioned above, the sample was synthesized from uncertified chemicals found in the laboratory. Thus, if the stock reagent were, in fact, impure $\mathrm{CeF}_{4}$ that actually contained a significant component of $\mathrm{CeF}_{3}$, there would, in fact, have been more $\mathrm{Ce}$ in the weighed sample and less $\mathrm{Cl}$ than expected on an absolute mass basis. This assessment is based upon the respective weights of the chemicals used to synthesize the sample. If the correct stoichiometric composition were included, the net effect would be to bring the weighed and PGAA data more into agreement. Thus, the PGAA data strongly suggest that stoichiometry may be determined for pure or simple 
mixtures of inorganic compounds. More work, especially with chemicals traceable to the United States National Institute for Standards and Technology (NIST) would be needed to confirm these assessments.

There is an additional point concerning the data obtained for the synthetic $\mathrm{CaF}_{2}, \mathrm{CeF}_{4}$ and $\mathrm{CeCl}_{3}\left(\mathrm{H}_{2} \mathrm{O}\right)_{\mathrm{x}}$ sample (Table 2). With the exception of a couple of elements like hydrogen (waters-ofhydration) and boron (impurity) which have only one prompt gamma line apiece, all other identified elements have multiple prompt gamma lines. This fact is significant for two reasons. First, multiple lines provide intrinsic cross-references not only to confirm qualitative identity but also to provide corroborative data that affirm the quantitative results (i.e., general agreement among the lines). Second, multiple PGAA lines are usually spread over a relatively wide energy range, in some cases, up to $\sim 11 \mathrm{MeV}$. Thus, one could potentially use the attenuation observed most notably for lower-energy lines and the relative lack of such effects on higher-energy lines to determine the approximate thickness of the attenuating medium. If one were to expand this reasoning to $4 \pi$ geometry (i.e., different detection angles in three-dimensional space), the evaluation of attenuative effects might afford insight into the approximate three-dimensional shape of an embedded target.

Very recently, new NAA measurements have begun with the LBNL neutron generator. The NAA spectrum of a $\mathrm{NaCl}$ sample irradiated for $15 \mathrm{~min}$ at $\sim 1.3 \times 10^{9}$ neutrons/second is shown in Figure 1. The decay of both the ${ }^{24} \mathrm{Na}(14.959 \mathrm{~h})$ and ${ }^{38} \mathrm{Cl}(37.24 \mathrm{~m})$ activation products are clearly seen and can be analyzed quantitatively to determine the stoichiometric ratio.

\section{Conclusion and Future Plans}

The data provided in Tables 1 and 2 demonstrate that PGAA is, indeed, a high-quality analytical process, and Figure 1 demonstrates NAA sensitivity with the LBNL neutron generator-based system. The impurity data and the data on the waters-of-hydration further indicate that more data may be obtained by one PGAA analysis than may be obtained by other currently accepted analytical methods. With a single irradiation, all significant elements may be detected using PGAA provided that the entire process is optimized; that is, neutron fluence is maximized and positioning of the target relative to the neutron source and the detector is configured for ideal geometry. The data also indicate a higher quality level than is usually reported by the suppliers of chemical reagents or standards because obtaining the level of analytical 
detail afforded by PGAA would require considerably more expenditure of time into alternative methodologies with the attendant costs and potential exposures. Furthermore, the general multi-line isotopic PGAA signatures provide a built-in system of checks and balances to corroborate results and add another layer of quality control to the analytical process. Agreement among different gamma rays provides added assurance of the quality of the reported results, while aberrations would indicate the presence of elemental cross contamination if results are high compared with the 'true' values or of attenuation if results are low compared with the 'true' values. This capability provides the analyst with more options for obtaining high-quality results regardless of any interferences or attenuative problems because the analyst may select lines devoid of such effects.

The identification of waters-of-hydration with PGAA provides a direct analytical method that no other nondestructive analytical technique affords. These waters-of-hydration would normally be determined inferentially on a massive sample by driving off the water, capturing the water on a pre-weighed dehydrating agent and determining the water gravimetrically by weight difference. In PGAA, the hydrogen and oxygen may be determined directly and independently, while the qualitative deduction of water arises from an evaluation of the atom $\%$ values (i.e., 2:1 ratio). Also, more significantly, there is no other nondestructive analytical technique that assays a given hydrogen atom directly. The most common alternative method is combustive analysis to form water and quantitation of the collected water by gravimetry.

From general documented records at LBNL, fluoride $\left(\mathrm{F}^{-}\right)$ion has apparently been used as part of the carrier substrate for much of the Laboratory's legacy inventory. The experiment conducted at the Budapest Reactor PGAA Facility using $\mathrm{CaF}_{2}, \mathrm{CeF}_{4}$ and $\mathrm{CeCl}_{3}\left(\mathrm{H}_{2} \mathrm{O}\right)_{\mathrm{x}}$ determined the $\mathrm{F}^{-}$by true PGAA, an experiment that will be repeated in some form at LBNL using the LBNL neutron generator. Also, as we have shown, LBNL can use short-lived neutron activation (NAA) as an adjunct to PGAA since the two methods are complementary. In certain cases (e.g., stable ${ }^{19} \mathrm{~F}$ and ${ }^{27} \mathrm{Al}$ ), the decay gammas from a short-lived activation product (e.g., ${ }^{20} \mathrm{~F}$ with a $\mathrm{t}_{1 / 2} \sim 11.03$ seconds and a single decay gamma of $1633.6 \mathrm{keV}$ with an absolute abundance $\sim 100 \%$ ) actually supersede, on the basis of sensitivity, the corresponding prompt capturegammas for the same isotopic moiety. Although the fluorine analysis on the synthetic sample described above was performed by PGAA, NAA for this element is better and can be performed simultaneously. For 
very short-lived NAA, the activation product attains equilibrium rapidly during the irradiation process and can be detected with the PGAA gammas. Thus, part of the LBNL PGAA research will include evaluation of short-lived NAA to determine its applicability for certain elements.

We are developing a complete PGAA design that optimizes the neutron intensity on target, the detector characteristics, shielding, and the overall system geometry. To increase neutron-capture crosssections further, 'cold' neutrons may be used. In this approach, thermal neutrons are cooled with any of various cryogenic moderators (e.g., liquid hydrogen) placed outside a (internally nickel-coated) guide tube. In accordance with the " $1 / v$ dependence" ${ }^{(8)}$, the capture cross-section increases as neutron temperature and, consequently, neutron velocity decreases, since $\sigma_{\mathrm{n}, \gamma} \propto 1 / v \propto 1 / \sqrt{T}$. Provided that the angle of impingement of the 'cold' neutrons is less than or equal to some specific critical angle - the largest for a single substance is for stable ${ }^{58} \mathrm{Ni}$ where $\theta_{\text {critical }}^{58_{N i}}=0.0173 \lambda\left\lfloor\theta_{\text {critical }}\right.$ (radians), $\left.\lambda(\mathrm{nm})\right\rfloor=0.47^{\circ} @ 0.4 \mathrm{~nm}{ }^{(9)}$ - the neutrons contained in the neutron guide are unattenuated for many meters and may be directed on target in a low-background counting area.

For PGAA to be accepted as a viable technique, not only must the quality of the overall process be demonstrated but also regulatory adoption of a general 'de minimus' level below which any irradiated material is not considered to be legally radioactive must be obtained. By definition, the PGAA/short-lived NAA process transmutes isotopes that may, in turn, be radioactive with variable half-lives. Short-lived activation products may be allowed to decay following irradiation. However, certain long-lived activation products may be produced like ${ }^{60} \mathrm{Co}$, although in very small radioactive quantities if the PGAA system is optimized Assuming a $10^{6} \mathrm{~ns}^{-1} \mathrm{~cm}^{-2}$ thermal neutron flux impinges on a target for one hour, no radionuclides with $\mathrm{t}_{1 / 2}>1$ year can be produced exceeding $80 \mathrm{~Bq} / \mathrm{cm}^{3}$. Both the United States Nuclear Regulatory Commission and the United States Department of Transportation have established such a 'de minimus' level at $74 \mathrm{~Bq} / \mathrm{gm}$ (2 nCi/gm). This value should suffice for PGAA/short-lived NAA but it would have to be adopted across the breadth of the United States Government. 


\section{Acknowledgments}

This work was supported by the Director, Office of Science, Office of Basic Energy Sciences, of the U.S.

Department of Energy under Contract No. DE-AC03-76SF00098.

\section{References}

(1) International Atomic Energy Agency (IAEA) Technical Report, to be published.

(2) K. DEBERTIN, R. G. HELMER, Gamma- and X-Ray Spectrometry with Semiconductor Detectors. North Holland, (1988) 92.

(3) E. BROWNE, R. B. FIRESTONE, Table of Radioactive Isotopes, John Wiley \& Sons, (1986).

(4) M. A. LONE, R. A. LEAVITT, D. A. HARRISON, Atomic Data and Nuclear Data Tables, 26 (1981) 511.

(5) D. L. PERRY, R. B. FIRESTONE, G. L. MOLNAR, ZS. REVAY, ZS. KASZTOVSZKY, R. C. GATTI, P. WILDE, J. Anal. At. Spect., 17 (2002) 32.

(6) G. A. ENGLISH, R. B. FIRESTONE, D. L. PERRY, J. REIJONEN, B. LUDEWIGT, K. N. LEUNG, G. GARABEDIAN, G. MOLNAR. ZS. REVAY, Nucl. Instr. Meth. B, to be published.

(7) D. L. PERRY, G. A. ENGLISH, R. B. FIRESTONE, K. N. LEUNG, G. GARABEDIAN, G. L. MOLNAR, ZS. REVAY, Nucl. Instr. Meth. B, to be published.

(8) G. FRIEDLANDER, J. W. KENNEDY, E. S. MACIAS, J. M. MILLER, Nuclear and Radiochemistry, $3^{\text {rd }}$ Ed., John Wiley \& Sons, (1981) 137.

(9) Z. B. ALFASSI (Ed.), Non-Destructive Elemental Analysis, $1^{\text {st }}$ Ed., Blackwell Science, (2001) 71 
Tables

Table 1: Atom \% Data from PGAA Analysis of Synthetic Legacy Sample

\begin{tabular}{|c|c|}
\hline Element & Atom (\%) \\
\hline \multicolumn{2}{|c|}{} \\
\hline $\mathrm{H}$ & 30.23 \\
\hline $\mathrm{B}$ & $8.52 \mathrm{E}-05$ \\
\hline $\mathrm{F}$ & 31.31 \\
\hline $\mathrm{Cl}$ & 6.26 \\
\hline $\mathrm{Ca}$ & 8.44 \\
\hline $\mathrm{Ce}$ & 8.57 \\
\hline $\mathrm{Fe}$ & $6.32 \mathrm{E}-02$ \\
\hline $\mathrm{Co}$ & $4.36 \mathrm{E}-03$ \\
\hline $\mathrm{Sm}$ & $5.14 \mathrm{E}-04$ \\
\hline $\mathrm{Gd}$ & $1.70 \mathrm{E}-04$ \\
\hline $\mathrm{Dy}$ & $3.89 \mathrm{E}-04$ \\
\hline $\mathrm{Er}$ & $3.33 \mathrm{E}-03$ \\
\hline $\mathrm{O}$ & 15.11 \\
\hline
\end{tabular}

Table 2: PGAA Results for Synthetic Legacy Sample

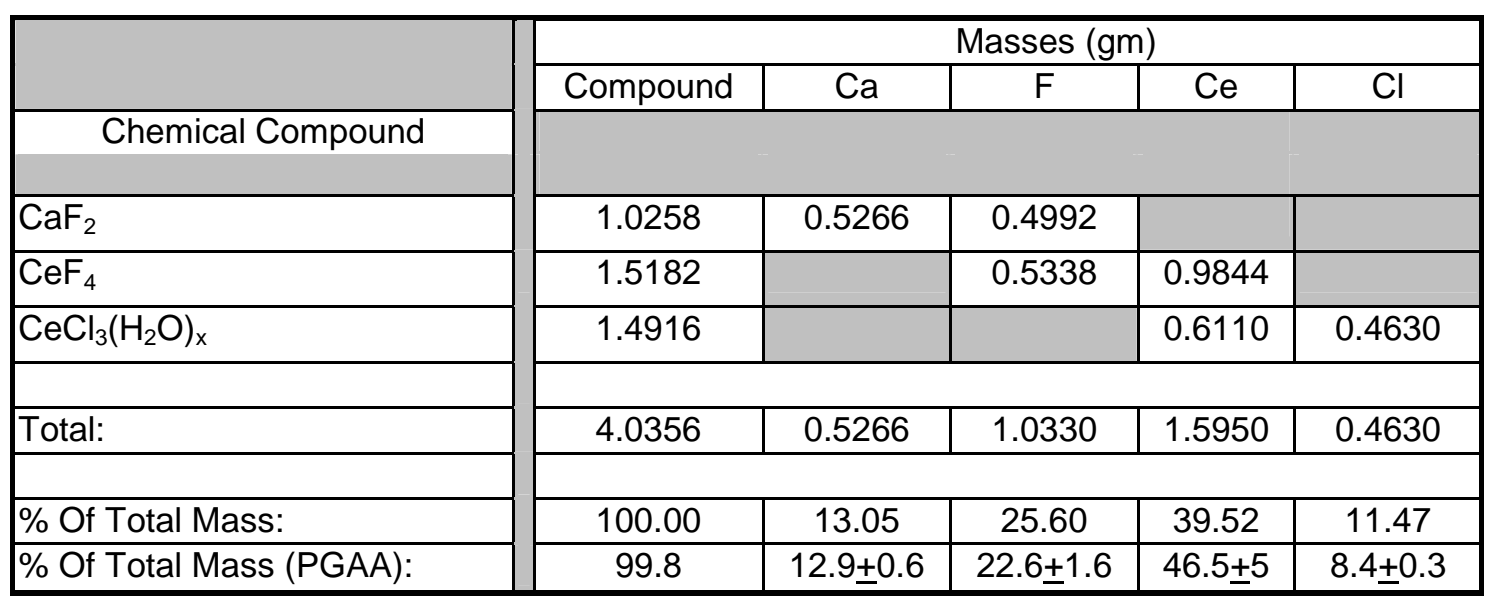


Figure

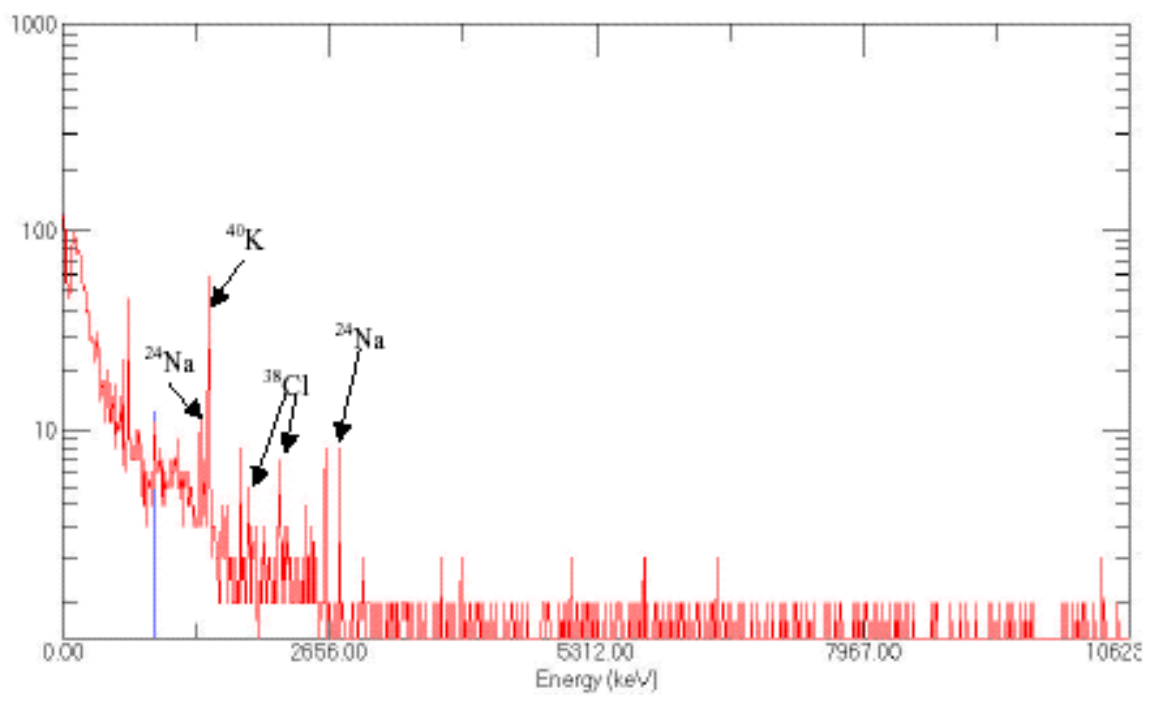

Figure 1. 10 minute count following 15-minute bombardment of $\mathrm{NaCl}$ sample. 